\section{DIAGNOSIS OF CHOLERA.}

TO THE EDITORS OF THE PROVINCIAL MEDICAL JOUBNAL.

Gentremen,-Having read the papers which have recently appeared in your Journal on the subject of malignant cholera, and being deeply impressed with the importance of the subject, I make no apology for troubling you with the following remarks, which, for the sake of brevity, I shall offer in the form of conclusions deduced from actual observation and reflection. That they may not be regarded as theoretical, it seems right to state that $I$ saw the disease at Musselburgh, Newbridge, and in the parish of Ratho, in Scotland, where I suffered from a very severe attack in my own person.

1. The term "cholera" appears to be quite inapplicable to the disease named "Asiatic or malignant cholera," whether we regard the symptoms or pathology of the disease. As this statement may be questioned by many, I will, as concisely as possible, mention the grounds upon which it rests, and which will suffice to show how little analogy there really is between the English disease, to which it is most justly applied, and that form which is to be considered in the sequel.

\section{ENGLISH CHOLERA.}

Diarrhœa and vomiting, accompanied with severe griping pain of abdomen, more especially about and below the umbilicus. The matter discharged in either may present an excess of acrid bile. The diarrhœa almost invariably preceding the vomiting, and both always persisting for some time, brief as it may be, in very bad cases, before sinking takes place. Urinary secretions, though diminished, never entirely suppressed. Cramps are common in the more severe cases of this disease; pyrexia frequent; pulse accelerated and wiry; thirst generally urgent. When the symptoms are not relieved the patient gradually sinks.

\section{ASIATIC DISEASE.}

Diarrhœa and vomiting may or may not be present. Diarrhœa not unfrequently exists for two or three days, but, being unattended by pain, attracts little or no attention. At the lapse of this time vomiting takes place, and collapse speedily ensues. In other cases collapse takes place without vomiting preceding it. It too frequently, however, happens that the premonitory state above described does not exist at all, but that the patient, without either diarrhœa or vomiting, is suddenly seized with prostration of power, and goes almost at once into the state of collapse, when diarrhœa, vomiting, cramps, burning heat at the epigastrium, occur. The pulse, at first slow and compressible, becomes small, and at last imperceptible; the eyes sunk in the orbits, and surrounded by dark areolæ; the cheeks hollow, and features sharp; tongue cold and clammy; voice reduced to a mere whisper; respiration not only feeble and slow, but on expiration imparting a sense of coldness to the face when brought near to the mouth of the patient; cramps almost incessant; the skin and integuments generally shrivelled and discolored, more especially about the fingers and toes, the nails of which are blue or livid. The craving for cold water is urgent, in consequence of the heat at the epigas- trium; this being the real cause, it can hardly be described as thirst, which appears to be a mixed sensation.

The above scanty glossary would appear almost sufficient to demonstrate the irreconcilable difference between the two diseases; but when the total suppression of urine, and the character of the evacuated matters are superadded, the difference is too manifest to require any comment. It is only necessary to add, that in the common or English disease I have never, even at the very last, witnessed a deficiency of bile, but, on the contrary, and invariably, a decided redundancy; whilst in the malignant disease, although the first discharges may be dark, and even scybalous, I have never seen a trace of bile until the disease had manifestly yielded to treatment, and the liver was again not only acting, but even overacting, probably under the influence of the calomel, to which such happy result must be ascribed, and which undue action, when not restrained, may prove fatal.

On the diagnosis of the disease it seems unnecessary to say one word, as I am aware of no disease with which it can for one moment be confounded.

As to the origin or causation of the disease, it will be admitted that it has, in its most capricious course, set aside the laws of infection and contagion, of epidemic and endemic influence, although it has seemed at times subservient to all of these agencies. It has also, from the situations in which it has prevailed, effectually divested itself of the characters of diseases originating in malaria-as ague, yellow fever, dysentery, \&c., confessedly do. That it is produced by some atmospheric cause, I entertain not a doubt, and that such atmospheric peculiarity is more immediately connected with electricity I feel equally con. vinced. This supposition is borne out by the fact of the great analogy existing between electricity and nervous influence; and the decided impression on most persons' nervous systems by that state of the atmosphere which precedes thunder storms, and against which but few persons are proof. If to this be added the sudden abstraction of nervous energy in the more severe cases of malignant cholera, this opinion will claim to itself more respect than may at first be awarded to it. That this impression is made directly on the cerebro-spinal system of nerves, and more particularly the respiratory, I firmly believe; and that the sensorium itself is only indirectly affected seems capable of demonstration. At present it is out of place to dilate upon this subject, but at an early opportunity it will, should it meet with your approval, afford me reai pleasure to do so.

2. The above view of the causation of cholera naturally leads to the following conclusion as to its pathology, and upon which we may consider the whole of the phenomena of this most extraordinary disease to depend. For the sake of perspicuity, no less than for that of avoiding prolixity, I will now, with every feeling of deference to the opinions of my professional brethren who may differ from me, state my belief, that cholera is the result of the sudden abstraction of nervous energy from the cerebro-spinal system of nerves, and that this is amply sufficient to explain the source of those symptoms which, taken collectively, constitute the disease, and may in fact be justly regarded as peculiar to it. Some of the more 
important of these symptoms I will now enumerate, and 1st, The altered state of the respiration.

2. The physical and chemical change in the constitution of the blood, which is abundantly proved both by ordinary examination and by chemical analysis.

3. The depressed and irregular action of the nervous system.

4. The suspension of the functions of the liver and kidneys.

The inference which I would draw from the coexistence of the above is, that in consequence of the sudden impression made on the nerves of respiration, the lungs do not adequately perform their office, the necessary result of which is the imperfect oxygenation of the blood, and its consequent unfitness for the purposes of life. Owing to this cause also, we have the impaired action of the heart and arteries, which are thereby deprived of their ordinary stimulus, to the very existence of which a due proportion of oxygen is admitted to be essential. The same cause explains the suspension of the secretions above mentioned, the organs being unable to act upon the morbid fluid circulating through them.

The nervous derangement I consider as dependent more immediately upon the effect produced upon the cerebellum and spinal nerves, if not confined to these; this opinion seems to acquire support from the fact, that in a vast majority of cases the function of the brain itself remains unimpaired, or at all events displays no irregularity up to the moment of dissolution. In this respect the phenomena of cholera display a very striking analogy to those attending the inhalation of poisonous gases, and more especially the carbonic acid gas. If further proofs of the correctness of the views which I have now ventured to express be required, these, I feel confident, will be met with by considering the effect of remedies on the disease.

From the length to which this paper has extended, I must now bring it to a somewhat abrupt termination, and were it not that the majority of the readers of your Journal are men both practical and experienced, I would feel considerable doubt as to the propriety of trusting so meagre an outline of so important a subject to their attention. This feeling, however, is much diminished by the spirit which has invariably pervaded the pages of your Journal.

I remain, Gentlemen,

Your most obedient servant, GEORGE Fife, M.D.

Newcastle-upon-Tyne,

Dec. 1, 1842.

\section{ON THE}

PRETENDED CURE OF CATARACT BY

\section{PRUSSIC ACID.}

By J. B. Estlin, F.L.S.,

Surgeon to the Bristol Dispensary for the Cure of Complaints in the Eye.

The readers of the Provincial Medical Journal are much indebted to Dr. Hastings (and so would the public be, had they access to his communications) for his exposure of the pretence of hydropathy to cure all kinds of disorders. A multitude will always be found in this country ready to become the dupes of those who boast, in an unqualified manner, of the efficacy of some particular remedy in the removal of every complaint; and it is not to be expected that persons will be wanting ever ready to turn this credulity to their own advantage, especially when they can persuade the public press to assist in the delusion. From this willingness to be deceived, Perkins's metallic tractors, animal magnetism, mustard seed, brandy and salt, homœopathy, hydropathy, \&c. \&c., have had their rise in popular estimation, and have had (or are having) their decline. I cannot admit that these vagaries do any injury to the regular practitioner; on the contrary, those in whose cases these professed remedies have proved inappropriate, and those who have been injured by them, are often driven to solicit his advice; and notwithstanding the continual discovery of remedies which, it is affected, will cure every complaint, and which should render the farther study of medicine a work of supererogation, there never was a period when our art was cultivated with more industry, when more minds of a high caste were devoted to its improvement, or when it held a more elevated rank in the estimation of the intelligent part of the community, than at the present moment.

A duty, however, appears to devolve upon the re. gular practitioner, and especially upon those who cannot be supposed to have any personal interest to serve in the matter, to prevent, as much as they can, a valuable remedy from getting into disrepute from its abuse, and to show the fallacy of ascribing curative powers to others totally destitute of the agency attributed to them. But is it not the case that those to whom the community have a right to look up for guidance in these matters, are too reluctant to come forward to disabuse the public mind on the subject of such pretended remedies?

Dr. Hastings has set a good example in this respect, and I am induced to follow it in illustrating by a case the folly of trusting to the promise which has been held out that cataract may be cured by the external application of the vapor of prussic acid, without operation.

To any educated surgeon it must be unnecessary to prove the futility of attempting the removal of a cataract-a hard tumor nearly in the centre of the eye, covered by a tough membranous capsule, ceasing probably from disease to have any organic relations with surrounding parts-by the application of a vapor to the cornea. The removal of opaque deposits upon the cornea itself by similar means I believe to be equally impracticable, but I now confine my remarks to cataract.

Though frequently asked by patients if I had heard of the powers of prussic acid in removing some of the most incurable or difficultly managed diseases of the eye, I was not aware of the monstrous pretensions that had been set up, and widely disseminated in newspapers and other periodicals not devoted to medical subjects, until the following case had come under my care :-

Mr. C., lately established in business as a draper, came to me from a town in Somersetshire on the 13th of October last to consult me respecting the state of his left eye. He was laboring under an acute attack of sclerotic inflammation, extending to the chambers 